\title{
Detección de pesticidas en el durazno (prunus pérsica) mediante una nariz electrónica
}

\section{Detection of pesticides in the peach (prunus pérsica) by an electronic nose}

\author{
Manuel Andrés Rivera-Guerrero' \\ Oscar Eduardo Gualdrón-Guerrero ${ }^{2}$ \\ Ivaldo Torres-Chávez ${ }^{3}$
}

Recibido: octubre 28 de 2019 Aceptado: diciembre 28 de 2019

\section{Resumen}

El presente trabajo tiene como objetivo el explorar las posibilidades de un sistema multisensorial, tipo nariz electrónica, para la detección de pesticidas organofosforados en el duraznero (Prunus pérsica), cultivado en el departamento de Norte de Santander, Colombia. Las pruebas se realizaron con una nariz electrónica conformada por 8 sensores químico-resistivos, y el método de procesamiento utilizado fue el análisis de componentes principales (PCA). Inicialmente se observó que los sensores reaccionan ante los compuestos volátiles presentes en el durazno, sin la presencia de pesticidas. Al agregar concentraciones conocidas de pesticidas al fruto, fue posible agrupar las muestras en cuatro grupos, así: sin pesticidas, 1, 2 y 3 ppm. Lo anterior indica que el sistema utilizado permite diferenciar las muestras puras de las que están contaminadas, además de agrupar los distintos rangos de concentración. Se concluye con la posibilidad de utilizar la nariz electrónica, en el control de calidad de productos agrícolas.

Palabras clave: nariz electrónica, durazno, pesticidas, PCA, organofosforados.

\begin{abstract}
The objective of this work is to explore the possibilities of a multisensory system, electronic nose type, for the detection of organophosphorus pesticides in the peach tree (Prunus persica), cultivated in the department of Norte de Santander, Colombia. The tests were performed with an electronic nose consisting of 8 chemical-resistive sensors, and the method of processing used was the principal component analysis (PCA). Initially it was observed that the sensors react to the volatile compounds present in the peach, without the presence of pesticides. By adding known concentrations of pesticides to the fruit, it was possible to group the samples into four groups, like this: without pesticides, 1, 2 and $3 \mathrm{ppm}$. The above indicates that the system used allows differentiating pure samples from contaminated ones, in addition to grouping the different concentration ranges. It concludes with the possibility of using the electronic nose, in the quality control of agricultural products.
\end{abstract}

Keywords: electronic nose, peach, pesticides, PCA, organophosphorus.

1 Ingeniero Químico, Universidad de Pamplona, Pamplona, Colombia. E-mail: manuel.rivera@unipamplona.edu.co. ORCID: 0000-0003-2840-0252

2 Ingeniero electrónico, Doctor en Ingeniería Electrónica, Universidad de Pamplona, Pamplona, Colombia. E-mail: oscar.gualdron@unipamplona. edu.co. ORCID: 0000-0002-7854-6842

3 Ingeniero electrónico, Doctor en Ingeniería Electrónica, Universidad de Pamplona, Pamplona, Colombia. E-mail: Ivaldo.torres@unipamplona. edu.co. ORCID: 0000-0003-4409-2616 


\section{Introducción}

El término pesticida, se utiliza para referirse a los productos fitosanitarios que se aplican a los cultivos para mantenerlos sanos y protegerlos de enfermedades y plagas (Ortiz-Villota, Romero-Morales \& Mesa-Rodríguez, 2018). Los pesticidas contienen sustancias activas, que pueden ser microorganismos o un producto químico, los cuales se pueden agrupar en familias, entre las cuales se incluyen: los organoclorados, organofosforados y los carbamatos (Maldonado-Obando \& Caballero-Pérez, 2016). Los productos fitosanitarios se clasifican según el peligro ambiental y para la salud humana. En cuanto a los riesgos para la salud, se tienen: corrosión (irritación de la piel), sensibilización respiratoria y de la piel, daño ocular grave, toxicidad aguda (oral, dérmica, inhalación), mutagenicidad, carcinogenicidad, así como toxicidad para la reproducción (Georgiadis et al., 2018).

Existen normativas para controlar el uso de los pesticidas, que indican cuales están prohibidos, así como la cantidad permitida de los que no lo están (Muñoz-Quesada et al., 2016). El uso indebido de los pesticidas causa problemas ambientales, por lo que se han adelantado estudios para monitorear y determinar los que perjudican el ambiente (Carazo-Rojas et al., 2018). Los pesticidas generan consecuencias en los seres humanos, tanto en las personas que los manipulan al añadírselos a los cultivos, así como en los consumidores, si los productos tienen cantidades fuera de los límites permitidos (Denghel \& Göen, 2018).

Los pesticidas que se usan para mejorar el rendimiento de los cultivos y para el manejo de plagas, son: los organoclorados (POC's), y organofosforados (POF's). Estos se emplean en una gran cantidad de alimentos, lo que ha llevado a realizar diversos estudios para establecer un mejor uso de los pesticidas, como en el caso del arroz (Berg \& Tam, 2018), y en el café (Reis, Fernandes, Lopes, Gorri, \& Alves, 2015). Sin embargo, en Colombia el uso de los pesticidas aún se lleva a cabo de manera inadecuada, sin el cumplimiento de la normatividad nacional e internacional, lo cual es un problema a la hora de llevar los productos a competir en los mercados extranjeros (González-Castro, Manzano-Durán \& García-Hoya, 2019).

Colombia es un país muy rico en diversidad de productos agrícolas, gracias a que presenta gran variedad de climas y pisos térmicos. Este es el caso del departamento de Norte de Santander, donde uno de esos productos es el durazno (prunus pérsica), que se produce en climas templados, dentro de la llamada fruticultura de clima frío (Yáñez-Urbina, Bautista-Rico \& Meléndez-Gelves, 2019). El durazno pertenece a la familia de los frutos caducifolios, y una gran cantidad de ellos se comercializan nacionalmente, incluso a veces solo regionalmente y con poco control en la cantidad de pesticidas que se le agrega, lo que hace que no cumpla con la reglamentación de Europa y Norteamérica (Miranda, Fischer, \& Carranza, 2013).

la Cromatografía de Gases es uno de los métodos analíticos empleados para determinar la cantidad de pesticidas en los alimentos. Este método permite separar componentes estrechamente relacionados en mezclas complejas, lo que lo diferencia de otras técnicas. El proceso se lleva a cabo desplazando la muestra con una fase móvil, usualmente un gas, la cual se hace pasar por una fase estacionaria, que se fija a una columna (Carrillo-Gómez, Durán-Acevedo \& García-Rico, 2019). Esta técnica necesita, en la mayoría de los casos, un pretratamiento de la muestra para eliminar interferencias indeseadas, por lo cual hay que conocer bien la composición de la muestra para saber qué pretratamiento y fase se utiliza (Skoog, Holler \& Nieman, 2001).

Este método tiene puntos positivos, como la alta sensibilidad para separar muestras complejas. No obstante, entre los puntos negativos están: los residuos de los solventes en el pretratamiento, es destructivo con la muestra, necesita de un operador capacitado; además, el equipo tiene un costo elevado, lo cual implica el análisis 
de la muestra en un laboratorio especializado (Paredes-Doig et al., 2019). Lo anterior implica que la cromatografía de gases, es una técnica que está lejos del alcance de los agricultores a mediana y pequeña escala.

El presente trabajo tiene como objetivo el explorar las posibilidades de un sistema multisensorial, tipo nariz electrónica, para la detección de pesticidas organofosforados en el duraznero (Prunus pérsica), cultivado en el departamento de Norte de Santander.

\section{Materiales y métodos}

\subsection{Nariz electrónica}

Una nariz electrónica es un instrumento que comprende una serie de sensores químicos electrónicos, con especificidad parcial, y un sistema de reconocimiento de patrones apropiado, capaz de reconocer olores simples o complejos (Gualdrón, Durán, Ortiz \& Araque, 2014). En forma coloquial, una nariz electrónica consiste en reemplazar las células receptoras de olor con sensores de gas, el procesamiento neuronal por una computadora y un software (Boeker, 2014).

La mayoría de las investigaciones se basa en la detección de los compuestos volátiles, característicos de cada muestra, los cuales determinan la elección de los sensores que incrementen la eficiencia en la nariz electrónica (Paredes-Doig et al., 2016). Luego de tener los sensores adecuados, se elige un método de reconocimiento de patrones que varía dependiendo del uso que se desee (Durán-Acevedo, Gualdrón-Guerrero \& Hernández-Ordóñez, 2014). Los métodos de reconocimiento de patrones pueden hacer una clasificación cualitativa o cuantitativa, y entre los más usados están: el análisis de componentes principales (PCA), los mínimos cuadrados parciales con análisis discriminante (PLS-DA) y la regresión de mínimos cuadrados parciales (PLSR) (Bona, Março \& Valderrama, 2018).
En este caso, se utilizó una nariz electrónica que se fabricó en la Universidad de Pamplona, como se observa en la figura 1. La nariz está formada por ocho sensores de gases tipo semiconductor TGS (Taguchis), de la casa japonesa Figaro Inc. Además, cuenta con una tarjeta de adquisición de datos marca National Instruments, lo que brinda una mayor precisión en la captura de la información arrojada por los sensores.

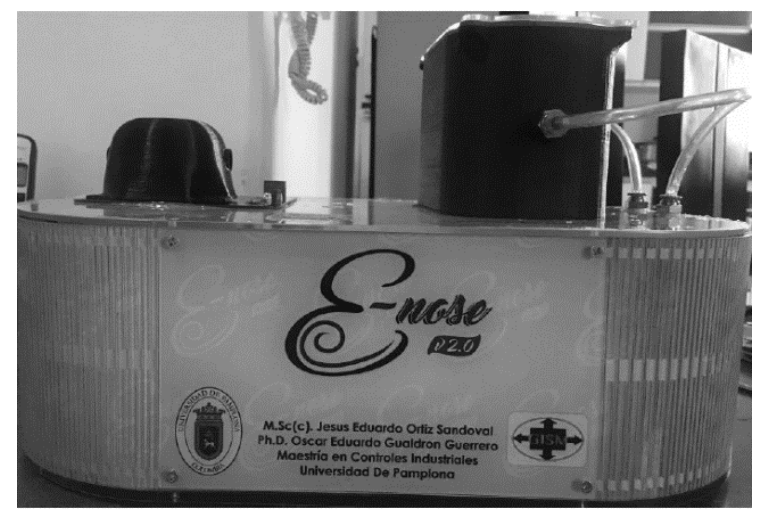

Figura 1. Sistema multisensorial (nariz electrónica).

El proceso de análisis de los frutos, a través de la nariz electrónica, cuenta con tres etapas, las cuales tienen unos tiempos característicos que permiten una toma de muestras óptima, como se indica a continuación:

La etapa de muestreo, donde se concentran los compuestos volátiles del fruto en una cámara para la posterior toma de datos (4 minutos).

Una segunda etapa que cuenta con la medición a través de los sensores químico-resistivos, los cuales responden a la presencia de los compuestos volátiles de la muestra (5 minutos).

La etapa de adquisición y procesamiento de las señales eléctricas que provienen de la matriz de sensores, para su posterior análisis (4 minutos). 


\subsection{Preparación de la muestra}

Las pruebas se llevaron a cabo con duraznos frescos, oriundos de los alrededores de la ciudad de Pamplona, Norte de Santander, Colombia. A este fruto se le agregó un pesticida organofosforado, que tiene como componente activo el clorpirifos, el cual se emplea comúnmente para el control de la mosca de la fruta, en concentraciones del rango de 1 a 3 ppm.

Las concentraciones del pesticida se prepararon a través de diluciones, utilizando balones aforados, donde se baja de 500000 ppm a 100ppm y luego a las concentraciones deseadas. Luego se impregnó el pesticida en los frutos, por inmersión, para cada concentración (1, 2 y 3ppm). Además, todas las pruebas se realizaron por triplicado.

\subsection{Adquisición y procesamiento de datos}

Para adquirir los datos y almacenar las señales de los sensores en el computador, se utilizó una tarjeta de alto rendimiento (DAQ 6009). Los datos se ingresaron en el software Bnose 2.0, que sirvió como interfaz gráfica. Este programa también permitió el manejo de los tiempos en el proceso de medición, el cual debe ser idéntico en todas las pruebas, para evitar inconvenientes en el análisis de los resultados.

El método de procesamiento utilizado fue el análisis de componentes principales (PCA), que funciona muy bien para aplicaciones que conlleven el uso de narices electrónicas, en las cuales las concentraciones de los volátiles no son elevadas y los sensores se comportan generalmente de manera lineal. El uso del algoritmo PCA en las narices electrónicas, se limita a representar gráficamente un conjunto de medidas para observar su posible agrupación (clusters), de medidas realizadas previamente (Durán-Acevedo, Gualdrón-Guerrero \& Hernández-Ordóñez, 2014).
El PCA es un método de reconocimiento de patrones no supervisado, donde la matriz $X$ se descompone en un producto de otras dos matrices: puntajes ( $\mathrm{T}$ ) y cargas (Pt). Para estos nuevos ejes, los componentes principales (PC) se calculan a través de algoritmos. La primera $\mathrm{PC}$, por lo general, pero no siempre, contiene la varianza máxima explicada del conjunto de datos. La segunda PC contiene una varianza explicada más baja que la PC1 y más alta que la PC3, y así sucesivamente. Por lo tanto, para elegir el número adecuado de PC, es posible evaluar el porcentaje de varianza explicada, la varianza explicada acumulativa o los valores propios (Bona et al., 2018).

\section{Resultados y discusión}

El primer paso de la investigación consistió en tomar mediciones del fruto sin pesticida, donde se observó que los sensores reconocieron los compuestos volátiles característicos de este, ver figura 2. Este aspecto fue primordial en la investigación, pues implica que los sensores que se encuentran en la nariz electrónica, son aptos para continuar con los análisis experimentales.

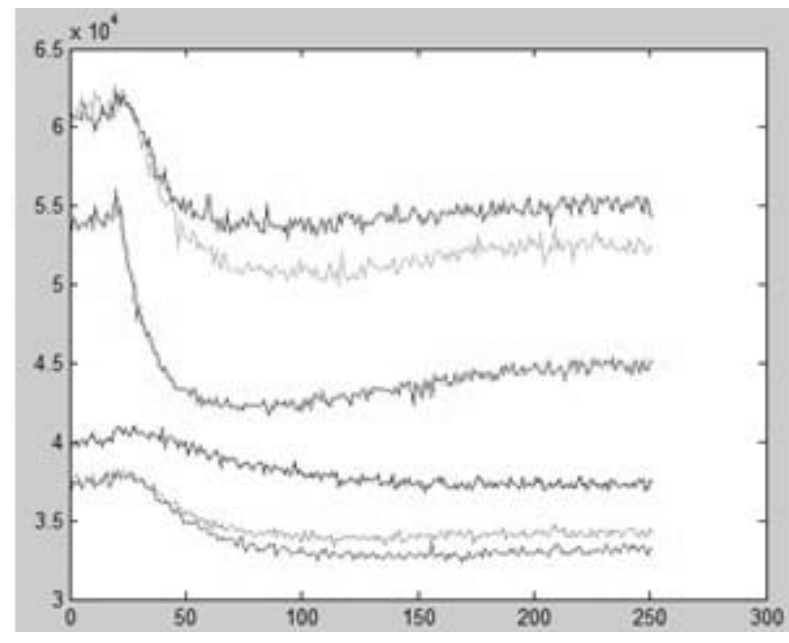

Figura 2. Respuesta de los sensores al fruto de durazno sin pesticidas.

Los valores obtenidos de la muestra indicaron cuáles sensores reaccionaban, donde se 
eliminaron dos que no presentaron cambios ante la presencia del fruto sin pesticidas. Los sensores que sí reaccionaron muestran una diferencia en sus picos de reacción, lo que conllevó a que el análisis estadístico diera un mejor resultado (ver figura 2).
Tomando los sensores que reaccionaron de manera satisfactoria al fruto sin pesticidas, se procedió a agregarle gradualmente una cantidad de pesticida de manera controlada, como se describió en la metodología. En la figura 3 se muestran los resultados de la detección realizada por la nariz electrónica.

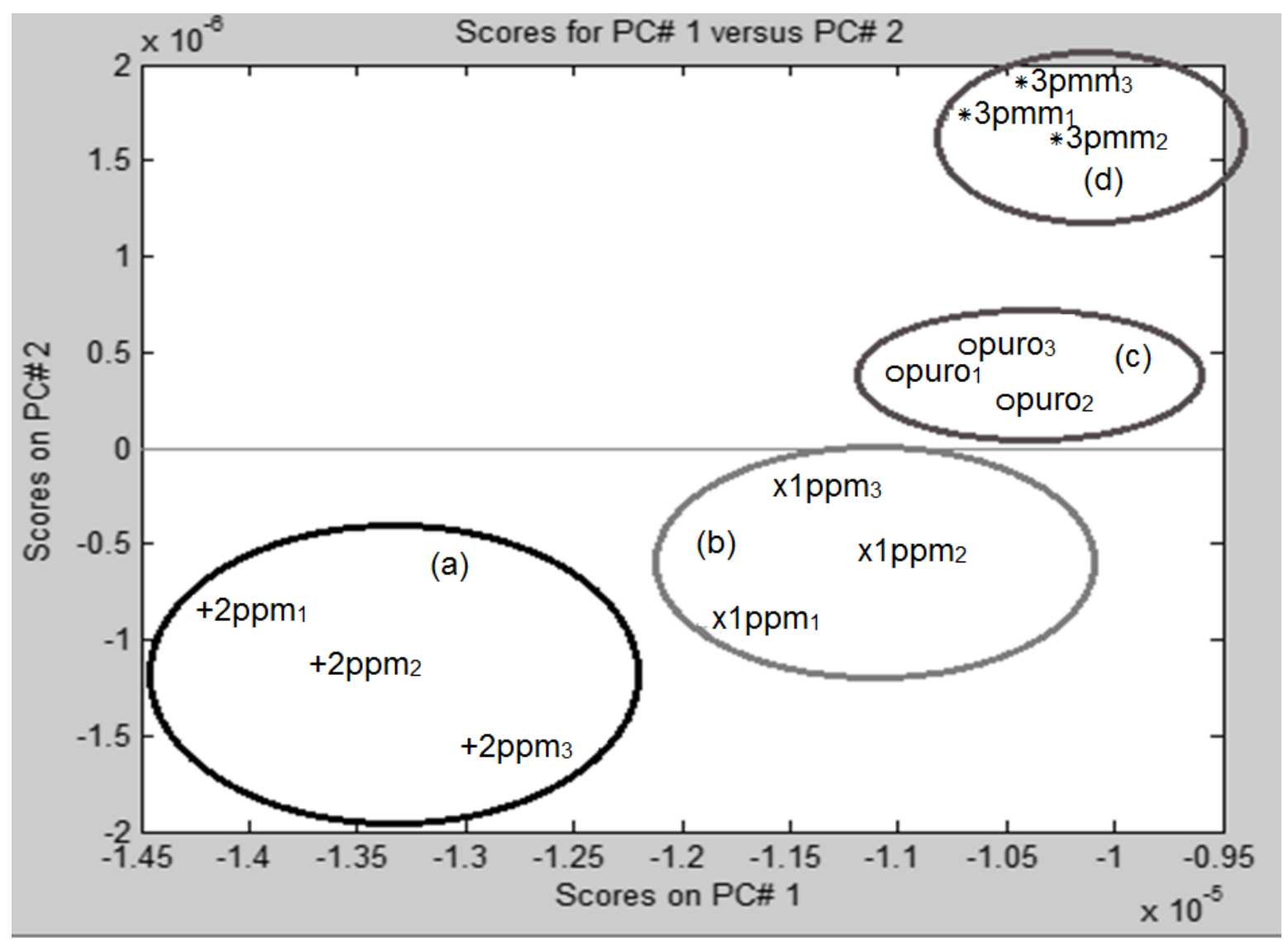

Figura 3. Discriminación de PCA del fruto puro (c), y con pesticida: 1 (b), 2 (a), y 3 ppm (d).

En el PCA se obtuvo una varianza de $99.8 \%$, con dos componentes principales (PC), lo cual indica que esta cantidad de componentes es la adecuada para brindar la información que tiene un valor significativo en el experimento. Esto debido a que si se aumenta la cantidad de PC, se puede incluir información no deseada. Una vez se tomaron estos dos PC, se continuó con la agrupación de todas las muestras.
En la figura 3, se observa la agrupación satisfactoria de las muestras en cuatro grupos, a saber: las muestras puras que se encuentran en el conjunto de datos (c), con una concentración de 1ppm agrupadas en (b) , 2 ppm en la parte inferior izquierda en el grupo (a) y finalmente 3 ppm en la esquina superior derecha en (d). Lo anterior indica que el sistema utilizado permite diferenciar las muestras puras de las que contienen pesticida, además de agrupar los distintos rangos de concentración. 
En este sentido, se puede decir que el método multisensorial utilizado, basado en una nariz electrónica, funciona de manera satisfactoria para evaluar la presencia de pesticidas y diferenciar la concentración en los frutos de durazno analizados. Resultados similares se encontraron al emplear una nariz electrónica para establecer el grado de madurez del tomate, así como para determinar la presencia del ajo en el desarrollo de productos funcionales (Durán-Acevedo et al., 2014; Ordóñez-Araque \& Barad, 2017). Lo anterior indica el potencial de estos dispositivos como alternativa de bajo costo, para su aplicación en el control de calidad de los cultivos de pequeños y medianos agricultores.

\section{Conclusiones}

Los sensores químico-resistivos empleados en esta investigación, son capaces de detectar los compuestos volátiles presentes en el durazno, lo que a su vez permitió efectuar la fase de detección de pesticidas. Lo anterior brinda la posibilidad de emplear este arreglo de sensores para diferenciar la etapa de maduración del fruto de durazno, así como para la clasificación de las distintas familias de este fruto.

Igualmente, los análisis de componentes principales, PCA, mostraron que es posible la detección de pesticidas organofosforados en los frutos de durazno, objetivo de esta investigación. Esto se pudo comprobar al añadir el pesticida al fruto, en concentraciones conocidas. En este sentido, se abre la posibilidad de cuantificar los pesticidas presentes en el durazno, empleando para ello sensores químico-resistivos de bajo costo.

\section{Referencias}

Berg, H., \& Tam, N. T. (2018). Decreased use of pesticides for increased yields of rice and fish-options for sustainable food production in the Mekong Delta. Science of The Total Environment, 619-620, 319-327. doi: https://doi.org/10.1016/j. scitotenv.2017.11.062
Boeker, P. (2014). On 'Electronic Nose' methodology. Sensors and Actuators B: Chemical, 204, 2-17. doi: https://doi.org/10.1016/j.snb.2014.07.087

Bona, E., Março, P. H., \& Valderrama, P. (2018). Chapter 4 - Chemometrics Applied to Food Control. In A. M. Holban \& A. M. Grumezescu (Eds.), Food Control and Biosecurity, 105-133: Academic Press.

Carazo-Rojas, E., Pérez-Rojas, G., Pérez-Villanueva, M., Chinchilla-Soto, C., Chin-Pampillo, J. S., Aguilar-Mora, P., \& Vryzas, Z. (2018). Pesticide monitoring and ecotoxicological risk assessment in surface water bodies and sediments of a tropical agro-ecosystem. Environmental Pollution, 241, 800-809. doi: https://doi.org/10.1016/j. envpol.2018.06.020

Carrillo-Gómez, J. K., Durán-Acevedo, C. M., \& García-Rico, R. O. (2019). Bacterial discrimination of drinking water through of an electronic nose and a volatiles extraction equipment. Revista Colombiana de Tecnologías de Avanzada, 1 (33), 155-165. doi: https://doi.org/10.24054/16927257. v33.n33.2019.3335

Denghel, H., \& Göen, T. (2018). Simultaneous assessment of phenolic metabolites in human urine for a specific biomonitoring of exposure to organophosphate and carbamate pesticides. Toxicology Letters, 298, 33-41. doi: https://doi. org/10.1016/j.toxlet.2018.07.048

Durán-Acevedo, C. M., Gualdrón-Guerrero, O. E., \& Hernández-Ordóñez, M. (2014). Nariz electrónica para determinar el índice de madurez del tomate de árbol (Cyphomandra Betacea Sendt). Ingeniería, Investigación y Tecnología, 15 (3), 351-362. doi: https://doi.org/10.1016/S1405-7743(14)70346-4

Georgiadis, N., Tsarouhas, K., Tsitsimpikou, C., Vardavas, A., Rezaee, R., Germanakis, I., \& Kouretas, D. (2018). Pesticides and cardiotoxicity. Where do we stand? Toxicology and Applied Pharmacology, 
353, 1-14. doi: https://doi.org/10.1016/j. taap.2018.06.004

González-Castro, Y., Manzano-Durán, O., \& GarcíaHoya, O. (2019). Puntos críticos de la cadena productiva de la mora (Rubus glaucus benth), en el municipio de Pamplona, Colombia. Revista de Investigación, Desarrollo e Innovación, 10 (1), 9-12. doi: 10.19053/20278306.v10.n1.2019.10008

Gualdrón, O., Durán, C., Ortiz, J., \& Araque, J. (2014). Implementation of a neural model in a hardware device(FPGA) for the classification of chemical compounds in a multisensory (E-nose). Revista Colombiana de Tecnologías de Avanzada, 2 (24), 127-133.

Maldonado-Obando, J., \& Caballero-Pérez, L. (2016). Estado del arte de los métodos para detección de pesticidas organoclorados en frutas. @ limentech, Ciencia y Tecnología Alimentaria, 14 (2), 104-130. doi: https://doi.org/10.24054/16927125. v2.n2.2016.2700

Miranda, D., Fischer, G., \& Carranza, C. (2013). Los frutales caducifolios en Colombia: Situación actual, caracterización de sistemas de producción y plan de desarrollo. Bogotá, Colombia: Sociedad Colombiana de Ciencias Hortícolas.

Muñoz-Quezada, M. T., Lucero, B., Iglesias, V., Muñoz, M. P., Achú, E., Cornejo, C., \& Brito, A. M. (2016). Plaguicidas organofosforados y efecto neuropsicológico y motor en la Región del Maule, Chile. Gaceta Sanitaria, 30 (3), 227-231. doi: https:// doi.org/10.1016/j.gaceta.2016.01.006

Ordóñez-Araque, R., \& Barat, J. M. (2017). Evaluación de un Sistema de Enmascaramiento de Olor de Muestras de Ajo, Mediante un Sistema de Nariz Electrónica. Revista Politécnica, 40 (1), 13-19. Recuperado de: http://scielo.senescyt. gob.ec/scielo.php?script=sci_arttext\&pi$d=S 1390-01292017000300013 \&$ Ing=es\&tlng=es.
Ortiz-Villota, M. T., Romero-Morales, M. A., \& Meza-Rodríguez, L. D. (2018). La biorremediación con microalgas (Spirulina máxima, Spirulina plantensis y Chlorella vulgaris) como alternativa para tratar la eutrofización de la laguna de Ubaque, Colombia. Revista de Investigación, Desarrollo e Innovación, 9 (1), 163-176. doi: https://doi. org/10.19053/20278306.v9.n1.2018.8153

Paredes-Doig, A. L., Sun-Kou, M. R., Picasso-Escobar, G., Doig-Camino, E., \& Comina, G. (2016). Implementación y evaluación de una nariz electrónica para la detección de alcoholes lineales. Revista Colombiana de Química, 45 (2), 12-18. doi: http://dx.doi.org/10.15446/ rev.colomb.quim. v45n2.60393

Paredes-Doig, A. L., Cárcamo, H., Hurtado-Cotillo, M., Sun-Kou, R., Doig-Camino, E., Picasso, G., Comina, G., \& La Rosa-Toro Gómez, A. (2019). Gas Sensors Modified with Zeolite $Y$ for Assessing Wine Aroma Compounds. Journal of Chemistry, 1. doi: https://doi.org/10.1155/2019/5283208

Reis, M. R. d., Fernandes, F. L., Lopes, E. A., Gorri, J. E. R., \& Alves, F. M. (2015). Chapter 26 - Pesticide Residues in Coffee Agroecosystems. In V. R. Preedy (Ed.), Coffee in Health and Disease Prevention, 235-244. San Diego: Academic Press.

Skoog, D., Holler, F., \& Nieman, T. (2001). Principios de análisis instrumental (5 ed.).

Yáñez-Urbina, L. F., Bautista-Rico, L. P., \& Meléndez-Gélvez, I. (2019). Genotoxic evaluation of durazno extracts prunus pérsica from the variety gran jarillo, cultivated the Chitagà, North of Santander . Revista Bistua, 17 (2), 126-136. 
